\title{
LOGARITHMIC BOUNDS ON SOBOLEV NORMS FOR TIME DEPENDENT LINEAR SCHRÖDINGER EQUATIONS
}

\author{
W.-M. WANG \\ Contents
}

1. Introduction and statement of the theorem

2. Periodic approximations and Floquet solutions

3. Some a priori estimates

4. Bounds on Sobolev norms 


\section{Introduction and statement of the theorem}

We consider the time dependent linear Schrödinger equation:

$$
i \frac{\partial}{\partial t} u=-\Delta u+V(x, t) u
$$

on $\mathbb{T} \stackrel{\text { def }}{=}[-\pi, \pi)$ with periodic boundary conditions. The potential $V$ is identified with a function on $\mathbb{R} \times \mathbb{R}$, periodic in $\mathbb{R}$ with period $2 \pi$. (To emphasize the time dependence, we write $V(x, t)$ for $V$.) We further assume that $V$ is real analytic in $(x, t)$ in a strip $D \stackrel{\text { def }}{=}(\mathbb{R}+i \rho)^{2}\left(|\rho|<\rho_{0}, \rho_{0}>0\right)$, real and bounded in $\mathbb{R}^{2}:\|V\|_{\infty, \mathbb{R}^{2}}<C<\infty$. We prove the following result:

Theorem. There exists $\varsigma>3$, such that for all $s>0$, there exists $C_{s}$, such that

$$
\|u(t)\|_{H^{s}} \leq C_{s}[\log (|t|+2)]^{\varsigma s}\|u(0)\|_{H^{s}},
$$

where $u(t)$ is the solution to (1.1) with the initial condition $u_{0} \in H^{s}$.

Remark. As we will see later, the theorem in fact extends to the class of potentials where the analyticity is replaced by some uniform estimates on the derivatives (e.g., Gevrey) and boundedness on $\mathbb{R}^{2}$ is weakened to logarithmic growth in $t$. The exponent $\varsigma$ will however depend on the Gevrey exponent. In this paper, we only state the theorem in the analytic case. Previously, it was proven in $[\mathrm{B} 2,3]$ that for $V \in C^{\infty}$,

$$
\|u(t)\|_{H^{s}} \leq C_{s, \epsilon}(|t|+1)^{\epsilon}\|u(0)\|_{H^{s}}
$$

for all $\epsilon>0$.

In $[\mathrm{N}]$, it was proven that for smooth time dependent potentials with certain random dependence on time, $\|u(t)\|_{H^{s}}$ is almost surely unbounded in time, which shows that the $\log$ in (1.2) is almost surely necessary. On the other hand, in [W], it was proven that for an explicit time periodic potential $\|u(t)\|_{H^{s}}$ remains bounded for all $t$. Clearly this belongs to the exceptional set from the point of view of random dependence in time. The present Theorem together with $[\mathrm{N}, \mathrm{W}]$ give a rather complete picture of time dependent linear Schrödinger equations on the circle.

The proof consists of making periodic in time approximations by replacing $V(x, t)$ with $V_{1}(x, t)$ which is periodic in $t$ with period $2 \pi T$ and $V_{1}(x, t)=V(x, t)$ for $|t| \leq T$. The dynamics of equation (1.1) is hence equivalent to the dynamics of

$$
i \frac{\partial}{\partial t} u=-\Delta u+V_{1}(x, t) u
$$

for $|t| \leq T$. This part of the strategy is similar to $[\mathrm{B} 2,3]$.

From Floquet theory, the dynamics of (1.3) can be reduced to the spectral theory of the corresponding Floquet operator

$$
H=\operatorname{diag}\left(\frac{n}{T}+j^{2}\right)+\hat{V}_{1} *
$$


on $\ell^{2}\left(\mathbb{Z}^{2}\right)$, where $n$ is the dual variable of $t, j$ the dual of $x$ and

$$
\hat{V}_{1}(j, n)=\int_{-\pi T}^{\pi T} \int_{-\pi}^{\pi} V_{1}(x, t) e^{-i j x} e^{-i \frac{n}{T} t} d x d t
$$

is the Fourier transform of $V_{1}$.

More specifically, let $\mathbb{T}_{T}=[-\pi T, \pi T)$ with periodic boundary conditions. For any initial datum $u_{0} \in L^{2}(\mathbb{T})$, we identify $u_{0}$ with $\check{u}_{0} \in L^{2}(\mathbb{T}) \times L^{2}\left(\mathbb{T}_{T}\right)$ as follows:

$$
\left\{\begin{array}{l}
\tilde{u}_{0}(j, 0)=\hat{u}_{0}(j) \\
\tilde{u}_{0}(j, n)=0, n \neq 0
\end{array}\right.
$$

where $\tilde{u}_{0}$ is the Fourier transform of $\check{u}_{0}$ and $\hat{u}_{0}$ that of $u_{0}$. All solutions to (1.3) can be written as linear superpositions of Floquet solutions, which up to a phase are inverse Fourier transforms of eigenfunctions to (1.4). Localization of eigenfunctions of (1.4) therefore leads to control over Sobolev norms of solutions to (1.3).

When $T$ is a fixed integer period, which is the resonant case as $\sigma(\Delta)=\left\{j^{2} \mid j \in\right.$ $\mathbb{Z}\} \subset \mathbb{N}$, it was proven in $[\mathrm{W}]$ that under appropriate conditions (1.4) has pure point spectrum with exponentially localized eigenfunctions. This in turn leads to

$$
\|u(t)\|_{H^{s}} \leq C_{s}\|u(0)\|_{H^{s}}
$$

for all $s>0$.

The main complication here is that $T$ is a variable, in fact $T \rightarrow \infty$. Using the identification (1.6) and the fact that for $|t| \leq T$ and large frequencies $|j|>J(T)$, the $H^{s}$ norm is essentially preserved, see (3.5) of Lemma 3.1, we construct approximate eigenfunctions to (1.4) by restricting $H$ to $H_{\Lambda}$ with $\Lambda=\{(j, n)|| j|\leq J(T)| n \mid, \leq$ $N(T)\}$ for approprate $J, N$ depending on $T$. This differs from [B2,3] and enables us to obtain logarithmic bounds.

We prove that these approximate eigenfunctions are localized in the proposition in sect. 2. The separation properties of the set $\left\{j^{2} \mid j \in \mathbb{Z}\right\}$ again plays an important role here as in $[\mathrm{W}]$. In sect. 4 , using the proposition and some a priori estimate for linear Schrödinger equations, we prove the theorem.

\section{Periodic approximations and Floquet solutions}

Let $u(t)$ be the solution to

$$
i \frac{\partial}{\partial t} u=-\Delta u+V(x, t) u
$$

with the initial condition $u_{0} \in H^{s}(0<s<\infty)$. We want to bound $\|u(t)\|_{H_{s}}$ as $t \rightarrow \infty$. (When $s=0$, the $L^{2}$ norm is conserved.) We therefore look at $(2.1)$ for $0 \leq|t| \leq \pi T, T \gg 1$, with the initial condition $u_{0}$. Let $\tilde{\phi} \in C_{0}^{\infty}[-\pi, \pi]$ be a (fixed) Gevrey function of order $\alpha$ :

$$
\max _{\tau \in[-\pi, \pi]}\left|\frac{\partial^{m} \tilde{\phi}(\tau)}{\partial \tau^{m}}\right| \leq C^{m+1}(m !)^{\alpha}, \quad 1<\alpha<\infty
$$


satisfying

$$
\left\{\begin{array}{l}
0 \leq \tilde{\phi} \leq 1 \\
\tilde{\phi}(\tau)=1,|\tau| \leq 1 \\
\tilde{\phi}(\tau)=0,|\tau| \geq \pi
\end{array}\right.
$$

(cf. $[\mathrm{Hö}])$.

Let

$$
\phi(t)=\tilde{\phi}\left(\frac{t}{T}\right)
$$

Define

$$
V_{1}(x, t)=\sum_{j \in \mathbb{Z}} V(x, t+2 \pi j T) \phi(t+2 \pi j T) .
$$

Then $V_{1}(x, t)$ is $2 \pi$ periodic in $x, 2 \pi T$ periodic in $t$, analytic in $x$, Gevrey in $t$ of order $\alpha,(1<\alpha<\infty)$.

$$
V_{1}(x, t)=V(x, t)
$$

for $0 \leq|t| \leq T$, and

$$
\left\|V_{1}\right\|_{\infty} \leq 2\|V\|_{\infty}
$$

So for $0 \leq|t| \leq T$, we can study instead the equation

$$
i \frac{\partial}{\partial t} u=-\Delta u+V_{1}(x, t) u \text {. }
$$

$V_{1}$ has the Fourier decomposition:

$$
V_{1}(x, t)=\sum_{j \in \mathbb{Z}, n \in \mathbb{Z}} \hat{V}_{1}(j, n) e^{i\left(j x+\frac{n}{T} t\right)},
$$

where

$$
\begin{gathered}
\left|\hat{V}_{1}(j, n)\right| \leq C e^{-c|j|}, \quad|j| \geq(\log T)^{\delta} \\
\leq C e^{-c\left|\frac{n}{T}\right|^{1 / \alpha}}, \quad|n| \geq T(\log T)^{\delta} \\
(T \gg 1,0<C, c, \delta<\infty) .
\end{gathered}
$$

Since we seek solutions to $(2.8)$ for finite time: $|t| \leq T$, it is convenient to replace $V_{1}$ by $V_{2}$ defined as

$$
V_{2}(x, t)=\sum_{\substack{|j| \leq(\log T)^{\sigma} \\|n| \leq T(\log T)^{\sigma}}} \hat{V}_{1}(j, n) e^{i\left(j x+\frac{n}{T} t\right)},
$$

where $\varsigma>\sigma>\alpha+\delta>1$. Using (2.10),

$$
\left\|V_{1}-V_{2}\right\|_{\infty} \leq e^{-(\log T)^{\sigma^{\prime} / \alpha}} \ll \frac{1}{T^{p}}
$$

provided $p<(\log T)^{\sigma^{\prime} / \alpha-1}(T \gg 1)$, where $1<\alpha+\delta<\sigma^{\prime}<\sigma$. For $|t| \leq T,(2.12)$ will permit us to use Floquet solutions to

$$
i \frac{\partial}{\partial t} u=-\Delta u+V_{2}(x, t) u
$$

in the approximation process in view of the following basic fact. 
Lemma 2.1. Let $\tilde{u}$ be an approximative solution of (2.8):

$$
\left(i \frac{\partial}{\partial t}+\Delta-V(x, t)\right) \tilde{u}=\eta
$$

with $\tilde{u}(t=0)=u_{0}$, where $\|\eta(t)\|_{L^{2}} \leq \epsilon$ for all $|t| \leq T$. Then the solution $u$ to (2.8) with $\tilde{u}(t=0)=u_{0}$ satisfies

$$
\|u(t)-\tilde{u}(t)\|_{L^{2}}<\epsilon|t| \leq \epsilon T
$$

for $|t| \leq T$.

Proof. Let $S(t)$ denote the flow of (2.8). This follows from the integral equation

$$
(\tilde{u}-u)(t)=i \int_{0}^{t} S(t) S(\tau)^{-1} \eta(\tau) d \tau
$$

and $\|S(t)\|_{L^{2} \rightarrow L^{2}}=1$.

Floquet solutions to (2.13)

Since $(2.13)$ is time periodic with period $2 \pi T$, any $L^{2}$ solution can be written as a linear superposition of Floquet solutions of the form $e^{i E t} \psi(x, t)$, where $\psi(x, t)$ is $2 \pi$ periodic in $x$ and $2 \pi T$ periodic in $t$ :

$$
\psi(x, t)=\sum_{(j, n) \in \mathbb{Z}^{2}} \hat{\psi}(j, n) e^{i\left(j x+\frac{n}{T} t\right)},
$$

$E$ is called the Floquet eigenvalue; $E, \hat{\psi}$ satisfy the eigenvalue equation:

$$
\begin{aligned}
H \hat{\psi} & =\left[\operatorname{diag}\left(\frac{n}{T}+j^{2}\right)+\hat{V}_{2} *\right] \hat{\psi} \\
& =E \hat{\psi}
\end{aligned}
$$

on $\ell^{2}\left(\mathbb{Z}^{2}\right)$, where $*$ denotes convolution:

$$
\begin{array}{cc}
\left(\hat{V}_{2} * \hat{\psi}\right)(j, n)=\sum_{\left(j^{\prime}, n^{\prime}\right) \in \mathbb{Z}^{2}} \hat{V}_{2}\left(j-j^{\prime}, n-n^{\prime}\right) \hat{\psi}\left(j^{\prime}, n^{\prime}\right), \\
\hat{V}_{2}(j, n)=\hat{V}_{1}(j, n) & \text { if }|j| \leq(\log T)^{\sigma} \text { and }|n| \leq T(\log T)^{\sigma}, \sigma>\alpha+\delta>1, \\
=0 & \text { otherwise, }
\end{array}
$$

and $\hat{V}_{1}$ satisfies $(2.10)$.

We identify the initial condition $\hat{u}_{0} \in \ell^{2}(\mathbb{Z})$ with $\tilde{u}_{0} \in \ell^{2}\left(\mathbb{Z}^{2}\right)$, where

$$
\left\{\begin{array}{l}
\tilde{u}_{0}(j, 0)=\hat{u}_{0}(j) \\
\tilde{u}_{0}(j, n)=0, \quad n \neq 0 .
\end{array}\right.
$$


Since we are only concerned about finite time: $|t| \leq T$, in view of $(2.12,2.17,2.18)$, Lemma 2.1, the a priori estimate (3.5) and some related estimates, which we will prove in sect. 3 (see Lemma 3.1), it is sufficient to solve the eigenvalue problem in (2.15) in a finite region

$$
\Lambda=\left\{(j, n) \in \mathbb{Z}^{2}|| j|\leq J(T),| n \mid \leq A T(\log T)^{\sigma}\right\},
$$

where $J(T)>T^{s}$ depending on $T$ and the Sobolev index $s, A>1$ as in the following proposition, $\sigma>\alpha+\delta>1$ as in (2.1).

For any subset $\mathcal{S} \subset \mathbb{Z}^{2}$, define $H_{\mathcal{S}}$ to be the restriction of $H$ to $\mathcal{S}$ :

$$
H_{\mathcal{S}}\left(n, j ; n^{\prime} j^{\prime}\right)= \begin{cases}H\left(n, j ; n^{\prime} j^{\prime}\right), & (n, j) \text { and }\left(n^{\prime}, j^{\prime}\right) \in \mathcal{S} \\ 0 & \text { otherwise. }\end{cases}
$$

We have the following estimates on eigenfunctions of $H_{\Lambda}$.

Proposition. Assume

$$
H_{\Lambda} \xi=E \xi, \quad\|\xi\|_{\ell^{2}(\Lambda)}=1 .
$$

Define

$$
\Omega_{0}=\left\{(j, n) \in \Lambda|| j \mid \leq 4 A(\log T)^{\sigma}\right\}, \quad(\sigma>\alpha+\delta>1)
$$

and for any $\left(j_{0}, n_{0}\right) \in \Lambda$, define

$\Omega^{\prime}\left(j_{0}, n_{0}\right)=\left\{(j, n) \in \Lambda|||j|-\left|j_{0}\right|\left|\leq(\log T)^{\sigma},\right| n-n_{0} \mid \leq T(\log T)^{\sigma}\right\},(\sigma>\alpha+\delta>1)$.

Then for all $\xi$ eigenfunctions of $H_{\Lambda}$ as in (2.21), $\xi$ satisfies either

$$
\begin{aligned}
\|\xi\|_{\ell^{2}\left(\Lambda \backslash \Omega_{0}\right)} & \leq e^{-(\log T)^{\left(\frac{\sigma^{\prime}-\delta}{\alpha}\right)}} \\
\text { or }\|\xi\|_{\ell^{2}\left(\Lambda \backslash \Omega^{\prime}\right)} & \leq e^{-(\log T)^{\left(\frac{\sigma^{\prime}-\delta}{\alpha}\right)}},\left(1<\alpha+\delta<\sigma^{\prime}<\sigma\right) .
\end{aligned}
$$

for some $\Omega^{\prime}=\Omega^{\prime}\left(j_{0}, n_{0}\right),\left(j_{0}, n_{0}\right) \in \Lambda$.

Proof. For any given $E$, we define the resonant set $\Omega$ such that if $(j, n) \in \Omega$, then

$$
\left|\frac{n}{T}+j^{2}-E\right| \leq(\log T)^{\sigma}, \quad(\sigma>\alpha+\delta>1) .
$$

So

$$
\left\|\left(H_{\Lambda \backslash \Omega}-E\right)^{-1}\right\| \leq \frac{1}{(\log T)^{\sigma}-\left\|V_{2}\right\|_{\infty}} \leq \frac{2}{(\log T)^{\sigma}},
$$

if

$$
\left\|V_{2}\right\|_{\infty}<\frac{1}{2}(\log T)^{\sigma}
$$

From (2.26), we have

$$
E-\frac{n}{T}-(\log T)^{\sigma} \leq j^{2} \leq E-\frac{n}{T}+(\log T)^{\sigma}, \quad(\sigma>\alpha+\delta>1)
$$

for $(j, n) \in \Omega$. We distinguish the following two cases: 
(i) $E \leq 5 A^{2}(\log T)^{2 \sigma}$

The less or equal part of (2.28) gives $|j| \leq 3 A(\log T)^{\sigma}$. So $\Omega \subset\{(j, n) \in \Lambda|| j \mid \leq$ $\left.3 A(\log T)^{\sigma}\right\}$. Define $B=\Lambda \backslash \Omega, B_{0}=\Lambda \backslash \Omega_{0}, B_{0} \subset B$. Let $P_{B}, P_{B_{0}}$ be projections onto the sets $B, B_{0}$.

Assume $\xi$ is an eigenfunction with eigenvalue $E \leq 5 A^{2}(\log T)^{2 \sigma}$. Then

$$
P_{B} \xi=-\left(H_{B}-E\right)^{-1} P_{B} \Gamma \xi
$$

where

$$
\Gamma=H_{\Lambda}-H_{B} \oplus H_{\Omega}
$$

So

$$
P_{B} \xi=-\left(H_{B}-E\right)^{-1} P_{B} \Gamma P_{\Omega} \xi
$$

Let

$$
\Gamma_{0}=H_{B}-H_{B_{0}} \oplus H_{B \backslash B_{0}} .
$$

Then

$$
\begin{aligned}
P_{B_{0}} \xi= & P_{B_{0}} P_{B} \xi \\
= & -P_{B_{0}}\left(H_{B_{0}}-E\right)^{-1} P_{B_{0}} \Gamma P_{\Omega} \xi \\
& +P_{B_{0}}\left(H_{B_{0}}-E\right)^{-1} \Gamma_{0}\left(H_{B}-E\right)^{-1} P_{B} \Gamma P_{\Omega} \xi,
\end{aligned}
$$

where we used $B_{0} \subset B$. Using $(2.27)$ on $\left(H_{B_{0}}-E\right)^{-1}$ and $\left(H_{B}-E\right)^{-1}$ and $(2.10$, $2.11,2.15)$ on $\Gamma, \Gamma_{0}$, we obtain

$$
\left\|P_{B_{0}} \xi\right\|_{\ell^{2}} \leq \frac{4 e^{-c(\log T)^{\sigma-\delta}}}{(\log T)^{2 \sigma}}<e^{-(\log T)^{\frac{\sigma^{\prime}-\delta}{\alpha}}} \quad\left(1<\alpha+\delta<\sigma^{\prime}<\sigma, T \gg 1\right),
$$

which is $(2.24)$.

(ii) $E>5 A^{2}(\log T)^{2 \sigma}$

The greater or equal part of (2.28) gives

$$
|j| \geq 2 A(\log T)^{\sigma} .
$$

So if there exist $(j, n),\left(j^{\prime}, n^{\prime}\right) \in \Omega \subset \Lambda,|j| \neq\left|j^{\prime}\right|$, then

$$
\left|\frac{n-n^{\prime}}{T}+j^{2}-j^{2}\right| \leq 2(\log T)^{\sigma}
$$

from (2.26). Using (2.35), this implies

$$
\begin{aligned}
\left|\frac{n-n^{\prime}}{T}\right| & \geq\left(|j|+\left|j^{\prime}\right|\right)\left(|j|-\left|j^{\prime}\right|\right)-2(\log T)^{\sigma} \\
& \geq(4 A-2)(\log T)^{\sigma} \\
& >2 A(\log T)^{\sigma}
\end{aligned}
$$

if $A>1$, which is a contradiction from the definition of $\Lambda$. So $|j|=\left|j^{\prime}\right|$ and

$$
\left|\frac{n-n^{\prime}}{T}\right| \leq 2(\log T)^{\sigma}<2 A(\log T)^{\sigma}
$$

for $A>1$, if both $(j, n),\left(j^{\prime}, n^{\prime}\right) \in \Omega$. (2.25) follows by using the same argument as in (2.29-2.34) with $\Omega^{\prime}$ replacing $\Omega_{0}$. 


\section{Some a priori estimates}

In this section, we collect some basic estimates on the flow of linear Schrödinger equations with smooth potentials, cf. [B1]. Since we will need estimates on $H^{s}$ norms for $s>0$ dependent on $T$, for completeness we also include their proofs, making explicit the dependence on $s$.

Let $S(t)$ be the flow of the linear Schrödinger equation in (2.1). Then $S(t)$ is unitary: $\|S(t)\|_{\ell^{2} \rightarrow \ell^{2}}=1$. Let $\Pi_{J}$ denote the Fourier multiplier defined as

$$
\begin{aligned}
\hat{\Pi}_{J} & =1, & & |j| \leq J / 2, \\
& =2(1-|j| / J), & & J / 2 \leq|j| \leq J, \\
& =0, & & |j|>J .
\end{aligned}
$$

Since $V$ is real analytic in $(x, t)$ and bounded in $D$, we have

$$
\left\|\frac{\partial^{m} V}{\partial x^{m}}\right\|_{\infty, \mathbb{T}} \leq C^{m+1} m !, \quad m=0,1, \ldots
$$

We have the following estimates on the $H^{s}$ norms:

\section{Lemma 3.1.}

$$
\begin{aligned}
& \|S(t)\|_{H^{s} \rightarrow H^{s}} \leq C^{s} s !\left(|t|^{s}+1\right), \\
& \left\|\left[V, \Pi_{J}\right]\right\|_{H^{s} \rightarrow H^{s}} \leq \frac{C s !}{J} \quad(J \gg 1), \\
& \left\|\left(I-\Pi_{J}\right) S(t)\right\|_{H^{s} \rightarrow H^{s}} \leq 1+\frac{\left(C^{s} s !\right)^{2}}{J}|t|^{s+1} \quad\left(J>|t|^{s}\right), \\
& \left\|\left[S(t), \Pi_{J}\right]\right\|_{H^{s} \rightarrow H^{s}} \leq \frac{\left(C^{s} s !\right)^{4}}{J}\left(|t|^{3 s+1}+1\right) \quad\left(J>|t|^{s}\right) .
\end{aligned}
$$

Remark. The same estimates hold for the flow of (2.8) as only the $x$-derivatives are involved.

Proof. Using (2.1),

$$
\begin{gathered}
\frac{\partial}{\partial t}\|u(t)\|_{H^{s}}^{2}=2 \operatorname{Re}\left(\frac{\partial^{s}}{\partial x^{s}} u(t), \frac{\partial^{s}}{\partial x^{s}} \frac{\partial}{\partial t} u(t)\right) \\
=2 \operatorname{Im}\left(\frac{\partial^{s}}{\partial x^{s}} u(t), \frac{\partial^{s}}{\partial x^{s}}(\Delta u+V u)\right) \\
=2 \operatorname{Im}\left(\frac{\partial^{s}}{\partial x^{s}} u(t), \sum_{\substack{\gamma+\beta=s \\
\gamma \geq 1}} \frac{\partial^{\gamma} V}{\partial x^{\gamma}} \frac{\partial^{\beta} u}{\partial x^{\beta}}\right) .
\end{gathered}
$$

It follows that

$$
\frac{\partial}{\partial t}\|u(t)\|_{H^{s}} \leq \sum_{\substack{\gamma+\beta=s \\ \gamma \geq 1 \\ 8}}\|u(t)\|_{H^{\beta}} C^{\gamma+1} \gamma !
$$


where we used (3.2).

Using interpolation:

$$
\|u(t)\|_{H^{s-1}} \leq\|u(t)\|_{H^{s}}^{\frac{s-1}{s}}\|u(t)\|_{L^{2}}^{\frac{1}{s}} \quad(s \geq 1),
$$

and more generally,

$$
\|u(t)\|_{H^{s-\gamma}} \leq\|u(t)\|_{H^{s}}^{\frac{s-\gamma}{s}}\|u(t)\|_{L^{2}}^{\frac{\gamma}{s}} \quad(s \geq \gamma) .
$$

Using (3.9) in (3.8), we have

$$
\begin{gathered}
\frac{\partial}{\partial t}\|u(t)\|_{H^{s}} \leq C^{2}\|u(t)\|_{H^{s}}^{1-\frac{1}{s}}\|u(t)\|_{L^{2}}^{\frac{1}{s}}+C^{3}\|u(t)\|_{H^{s}}^{1-\frac{2}{s}}\|u(t)\|_{L^{2}}^{\frac{2}{s}}+\cdots \\
+C^{\gamma+1} \gamma !\|u(t)\|_{H^{s}}^{1-\frac{\gamma}{s}}\|u(t)\|_{L^{2}}^{\frac{\gamma}{s}}+\cdots+C^{s+1} s !\|u(t)\|_{L^{2}}
\end{gathered}
$$

Since

$$
\frac{s}{\gamma}\|u(t)\|_{H^{s}}^{1-\frac{\gamma}{s}} \frac{\partial}{\partial t}\|u(t)\|_{H^{s}}^{\frac{\gamma}{s}}=\frac{\partial}{\partial t}\|u(t)\|_{H^{s}} \quad(1 \leq \gamma \leq s),
$$

we obtain from (3.10),

$$
\|u(t)\|_{H^{s}} \leq C^{s} s !\left(|t|^{s}+1\right)\left\|u_{0}\right\|_{H^{s}} .
$$

Hence

$$
\|S(t)\|_{H^{s} \rightarrow H^{s}} \leq C^{s} s !\left(|t|^{s}+1\right) .
$$

To prove (3.4), it is more convenient to work with the Fourier variables $j$ dual to $x$. Let $\hat{\hat{V}}$ be the partial Fourier transform with respect to $x$. we have

$$
\left.\left[V, \Pi_{J}\right] \hat{(} j, j^{\prime}\right)=\hat{\hat{V}}\left(j-j^{\prime}\right)\left(\hat{\Pi}_{J}\left(j^{\prime}\right)-\hat{\Pi}_{J}(j)\right),
$$

where $\hat{\Pi}_{J}$ is defined in (3.1). Since $V$ is analytic, periodic in $x$ and $|V(x, t)|<C$ for all $t$,

$$
\begin{aligned}
& \left|\hat{\hat{V}}\left(j-j^{\prime}\right)\right| \leq C e^{-c\left|j-j^{\prime}\right|}, \\
& \text { and from (3.1) } \\
& \begin{aligned}
\left|\hat{\Pi}_{J}\left(j^{\prime}\right)-\hat{\Pi}_{J}(j)\right| & \leq 1, & & \left|j-j^{\prime}\right| \geq J / 2, \\
& \leq \frac{2}{J}\left|j-j^{\prime}\right|, & & \left|j-j^{\prime}\right|<J / 2 .
\end{aligned}
\end{aligned}
$$

Using (3.15), we have

$$
\begin{aligned}
\left.\mid\left[V, \Pi_{J}\right] \hat{(j} j, j^{\prime}\right) \mid & \leq C e^{-c\left|j-j^{\prime}\right|}, & & \left|j-j^{\prime}\right| \geq J / 2 \\
& \leq \frac{2 C}{J}\left|j-j^{\prime}\right| e^{-c\left|j-j^{\prime}\right|}, & & \left|j-j^{\prime}\right|<J / 2
\end{aligned}
$$

From Schur's lemma, we then obtain (3.4). 
To prove $(3.5)$, we proceed similarly to the proof of (3.3). We have

$$
\begin{aligned}
\frac{\partial}{\partial t}\left\|\left(I-\Pi_{J}\right) u(t)\right\|_{H^{s}}^{2}= & 2 \operatorname{Im}\left(\left(I-\Pi_{J}\right) \frac{\partial^{s}}{\partial x^{s}} u(t),\left(I-\Pi_{J}\right) V \frac{\partial^{s}}{\partial x^{s}} u(t)\right) \\
& +2 \operatorname{Im}\left(\left(I-\Pi_{J}\right) \frac{\partial^{s}}{\partial x^{s}} u(t),\left(I-\Pi_{J}\right) \sum_{\substack{\gamma+\beta=s \\
\gamma \geq 1}} \frac{\partial^{\gamma} V}{\partial x^{\gamma}} \frac{\partial^{\beta} u}{\partial x^{\beta}}\right) .
\end{aligned}
$$

So

$$
\begin{aligned}
& \frac{\partial}{\partial t}\left\|\left(I-\Pi_{J}\right) u(t)\right\|_{H^{s}} \\
\leq & {\left[V, \Pi_{J}\right]\|u(t)\|_{H^{s}}+\left[\frac{\partial V}{\partial x}, \Pi_{J}\right]\|u(t)\|_{H^{s-1}}+C\left\|\left(I-\Pi_{J}\right) u(t)\right\|_{H^{s-1}} } \\
& +\cdots+\left[\frac{\partial^{\gamma} V}{\partial x^{\gamma}}, \Pi_{J}\right]\|u(t)\|_{H^{s-\gamma}}+C^{\gamma+1} \gamma !\left\|\left(I-\Pi_{J}\right) u(t)\right\|_{H^{s-\gamma}} \\
& +\cdots+\left[\frac{\partial^{s} V}{\partial x^{s}}, \Pi_{J}\right]\|u(t)\|_{L^{2}}+C^{s+1} s !\left\|\left(I-\Pi_{J}\right) u(t)\right\|_{L^{2}}
\end{aligned}
$$

Using

$$
\begin{aligned}
& \left\|\left[\frac{\partial^{\gamma} V}{\partial x^{\gamma}}, \Pi_{J}\right]\right\|_{H^{s-\gamma} \rightarrow H^{s-\gamma}} \leq \frac{C s !}{J} \\
& \left\|\left(I-\Pi_{J}\right) u(t)\right\|_{H^{s-\gamma}} \leq \frac{1}{J^{\gamma}}\|u(t)\|_{H^{s}}
\end{aligned}
$$

and (3.9) in (3.18) and integrating over $t$, we obtain (3.5).

To prove (3.6), assume $u$ is a solution to (2.1)

$$
i \frac{\partial}{\partial t} u+\Delta u-V(x, t) u=0
$$

then

$$
\left(i \frac{\partial}{\partial t}+\Delta\right) \Pi_{J} u-V\left(\Pi_{J} u\right)=-\left[V, \Pi_{J}\right] u
$$

From Lemma 2.1

$$
\begin{aligned}
& {\left[S(t), \Pi_{J}\right] u_{0} } \\
= & S(t) \Pi_{J} u_{0}-\Pi_{J} S(t) u_{0} \\
= & -i \int_{0}^{t} S(t) S(\tau)^{-1}\left[V, \Pi_{J}\right] u(\tau) d \tau
\end{aligned}
$$

Using $(3.3,3.4)$ in $(3.22)$, we obtain $(3.6)$.

\section{Bounds on Sobolev norms}

Let $u_{0} \in H^{s}$ be an initial datum, normalized so that $\left\|u_{0}\right\|_{H^{s}}=1$. We assume $0<s \leq \log T$, cf. (4.49). Let

$$
J=T^{10 s}
$$


Then from (3.5)

$$
\begin{aligned}
\left\|S(0, t) u_{0}\right\|_{H^{s}} & =\left\|\Pi_{J / 4} S(0, t) u_{0}\right\|_{H^{s}}+\left\|\left(1-\Pi_{J / 4}\right) S(0, t) u_{0}\right\|_{H^{s}} \\
& \leq\left\|\Pi_{J / 4} S(0, t) u_{0}\right\|_{H^{s}}+2\left\|u_{0}\right\|_{H^{s}}
\end{aligned}
$$

In view of the proposition and (4.2), let

$$
J_{0}=4 A(\log T)^{\sigma} \quad(A>1, \sigma>\alpha+\delta>1) .
$$

We make the following decomposition:

$$
\begin{aligned}
\left\|\Pi_{J / 4} S(0, t) u_{0}\right\|_{H^{s}} & \leq\left\|\Pi_{J / 4} S(0, t) \Pi_{2 J_{0}} u_{0}\right\|_{H^{s}} \\
& +\left\|\Pi_{J / 4} S(0, t)\left(\Pi_{J / 2}-\Pi_{2 J_{0}}\right) u_{0}\right\|_{H^{s}} \\
& +\left\|\Pi_{J / 4} S(0, t)\left(I-\Pi_{J / 2}\right) u_{0}\right\|_{H^{s}}
\end{aligned}
$$

(4.6) can be estimated using (3.5):

$$
\begin{aligned}
\left\|\Pi_{J / 4} S(0, t)\left(I-\Pi_{J / 2}\right) u_{0}\right\|_{H^{s}} & \leq\left\|\left[S(0, t), \Pi_{J / 2}\right] u_{0}\right\|_{H^{s}} \\
& \leq 2 \frac{\left(C^{s} s !\right)^{4}}{J}\left(1+T^{3 s+1}\right)\left\|u_{0}\right\|_{H^{s}} \\
& <1
\end{aligned}
$$

To estimate (4.5), we use the following

Lemma 4.1. Let $\phi$ be such that

$$
\text { supp } \hat{\phi} \subseteq\left[-J / 2,-2 J_{0}\right] \cup\left[2 J_{0}, J / 2\right] \text {, }
$$

then

$$
\left\|\Pi_{J / 4} S(t) \phi\right\|_{H^{s}} \leq C^{s}\|\phi\|_{H^{s}} .
$$

Proof. We identify $\hat{\phi}$ with $\tilde{\phi}$ defined as

$$
\left\{\begin{array}{l}
\tilde{\phi}(j, 0)=\hat{\phi}(j) \\
\tilde{\phi}(j, n)=0, \quad n \neq 0
\end{array}\right.
$$

supp $\tilde{\phi} \subset \Lambda$, where $\Lambda$ is defined in (2.19). $\tilde{\phi} \in \ell^{2}(\Lambda)$. So we can expand $\tilde{\phi}$ using the eigenfunctions $\xi$ of $H_{\Lambda}$ :

$$
\tilde{\phi}=\sum(\tilde{\phi}, \xi) \xi
$$

Let $\chi_{S}$ be the characteristic function of the set $S$ :

$$
\left.\chi_{S}\right|_{S}=1,\left.\quad \chi_{S}\right|_{\Lambda \backslash S}=0
$$


For an eigenfunction $\xi$ satisfying (2.25), let

$$
\xi^{\prime}=\chi_{\Omega^{\prime}} \xi, \quad Q=\left\{\xi^{\prime} \mid \xi \text { satisfies }(2.25)\right\} .
$$

Using $(4.8,4.10,2.24,2.25)$ in $(4.11)$, we have

$$
\left\|\tilde{\phi}-\sum_{\xi^{\prime} \in Q}\left(\tilde{\phi}, \xi^{\prime}\right) \xi^{\prime}\right\|_{\ell^{2}(\Lambda)} \leq \mathcal{O}\left(e^{-(\log T)^{\left(\frac{\sigma^{\prime}-\delta}{\alpha}\right)}} \sqrt{|\Lambda|}\|\hat{\phi}\|_{\ell^{2}}\right) .
$$

Since $|\Lambda| \leq T^{10 s+2}$ from $(2.19,4.1)$ and $0<s \leq \log T$, we have

$$
\left\|\tilde{\phi}-\sum_{\xi^{\prime} \in Q}\left(\tilde{\phi}, \xi^{\prime}\right) \xi^{\prime}\right\|_{\ell^{2}(\Lambda)} \leq e^{-\frac{2}{3}(\log T)^{\left(\frac{\sigma^{\prime}-\delta}{\alpha}\right)}}
$$

assuming

$$
\sigma^{\prime}>2 \alpha+\delta>2
$$

From $(4.13,2.25), \xi^{\prime}$ is an approximate eigenfunction of $H_{\Lambda}$ :

$$
\begin{aligned}
\left\|\left(H_{\Lambda}-E\right) \xi^{\prime}\right\|_{\ell^{2}(\Lambda)} & \leq\left\|\left(H_{\Lambda}-E\right)\right\|_{\ell^{2} \rightarrow \ell^{2}}\|\xi\|_{\ell^{2}\left(\Lambda \backslash \Omega^{\prime}\right)} \\
& =e^{-\frac{2}{3}(\log T)^{\left(\frac{\sigma^{\prime}-\delta}{\alpha}\right)}}, \quad\left(\sigma^{\prime}>2 \alpha+\delta>2\right) .
\end{aligned}
$$

Hence $\xi^{\prime}$ is an approximate eigenfunction of $\tilde{H}$ :

$$
\tilde{H}^{\text {def }} \stackrel{\operatorname{diag}}{=}\left(j^{2}+\frac{n}{T}\right)+\hat{V}_{1} *=H+\left(\hat{V}_{1}-\hat{V}_{2}\right) *,
$$

where $H$ as defined in $(2.15), \hat{V}_{1}$ is defined in $(2.5,2.9)$ satisfying $(2.10)$. This is because

$$
(\tilde{H}-E) \xi^{\prime}=\left(H_{\Lambda}-E\right) \xi^{\prime}+\Gamma \xi^{\prime}+\left(\hat{V}_{2}-\hat{V}_{1}\right) * \xi^{\prime},
$$

where $H_{\Lambda}$ as defined in $(2.20,2.19)$,

$$
\Gamma=H-H_{\Lambda} \oplus H_{\Lambda^{c}}
$$

and

$$
\left\|(\tilde{H}-E) \xi^{\prime}\right\|_{\ell^{2}} \leq 2 e^{-\frac{2}{3}(\log T)^{\left(\frac{\sigma^{\prime}-\delta}{\alpha}\right)}} \quad\left(\sigma^{\prime}>2 \alpha+\delta>2\right),
$$

using $(4.17,2.12)$.

Define

$$
\check{\xi}(x, t)=e^{i E t} \sum_{(j, n) \in \Omega^{\prime}} \xi^{\prime}(j, n) e^{i\left(j x+\frac{n}{T} t\right)} .
$$

From (4.18), $\check{\xi}$ is an approximate Floquet solution of (2.8) satisfying

$$
\left(i \frac{\partial}{\partial t}+\Delta-V_{1}\right) \check{\xi}=e^{-\frac{2}{3}(\log T)^{\left(\frac{\sigma^{\prime}-\delta}{\alpha}\right)}} \quad\left(\sigma^{\prime}>2 \alpha+\delta>2\right) .
$$


Let $S(t)$ be the flow for equation (2.8), using Lemma 2.1

$$
\|\check{\xi}(t)-S(t) \check{\xi}(0)\|_{2} \leq T e^{-\frac{2}{3}(\log T)^{\left(\frac{\sigma^{\prime}-\delta}{\alpha}\right)}} .
$$

Because of localization properties of $\xi^{\prime}$ in (2.25), we will have good control over $\|\check{\xi}(t)\|_{H^{s}}($ see $(4.28-4.37))$.

In view of (4.24), we express $\phi$ as an approximate linear combination of $\check{\xi}(0)$ as follows. Taking the inverse Fourier transform of the expression under the norm sign in (4.14), we have equivalently

$$
\left\|\tilde{\phi}(x, \theta)-\sum_{\xi^{\prime} \in Q}\left(\tilde{\phi}, \xi^{\prime}\right) \sum_{(j, n) \in \Omega^{\prime}} \xi^{\prime}(j, n) e^{i\left(j x+\frac{n}{T} \theta\right)}\right\|_{L^{2}\left(\mathbb{T} \times \mathbb{T}_{T}\right)} \leq e^{-\frac{2}{3}(\log T)^{\left(\frac{\sigma^{\prime}-\delta}{\alpha}\right)}},
$$

where $\mathbb{T}$ denotes $[-\pi, \pi)$ with periodic boundary conditions and $\mathbb{T}_{T}$ denotes $[-\pi T, \pi T)$ with periodic boundary conditions.

So $\phi(x) \stackrel{\text { def }}{=} \phi(x, 0)$ as a function on $L^{2}(\mathbb{T})$ satisfies

$$
\begin{aligned}
& \left\|\phi(x)-\sum_{\xi^{\prime} \in Q}\left(\tilde{\phi}, \xi^{\prime}\right) \sum_{(j, n) \in \Omega^{\prime}} \xi^{\prime}(j, n) e^{i j x}\right\|_{L^{2}(\mathbb{T})} \\
\leq & T^{1 / 2}(\log T)^{\sigma / 2} e^{-\frac{2}{3}(\log T)^{\left(\frac{\sigma^{\prime}-\delta}{\alpha}\right)}} \\
\leq & e^{-\frac{1}{2}(\log T)^{\left(\frac{\sigma^{\prime}-\delta}{\alpha}\right)}},\left(\sigma>\sigma^{\prime}>2 \alpha+\delta>2\right) .
\end{aligned}
$$

Therefore for $|t| \leq T,(4.24,4.26)$ give

$$
\begin{aligned}
& \left\|S(t) \phi-\sum_{\xi^{\prime} \in Q}\left(\tilde{\phi}, \xi^{\prime}\right) \check{\xi}(t)\right\|_{L^{2}(\mathbb{T})} \\
\leq & e^{-\frac{1}{2}(\log T)^{\left(\frac{\sigma^{\prime}-\delta}{\alpha}\right)}}+\sum_{\xi^{\prime} \in Q} \mid\left(\tilde{\phi}, \xi^{\prime}\right)\|\check{\xi}(t)-S(t) \check{\xi}(0)\|_{L^{2}(\mathbb{T})} \\
\leq & e^{-\frac{1}{2}(\log T)^{\left(\frac{\sigma^{\prime}-\delta}{\alpha}\right)}}+|\Lambda| T e^{-\frac{2}{3}(\log T)^{\left(\frac{\sigma^{\prime}-\delta}{\alpha}\right)}} \\
\leq & 2 e^{-\frac{1}{2}(\log T)^{\left(\frac{\sigma^{\prime}-\delta}{\alpha}\right)}} \quad\left(\sigma^{\prime}>2 \alpha+\delta>2\right),
\end{aligned}
$$

where we used $(4.1,2.19)$.

So we only need to estimate $\left\|\sum_{\xi^{\prime} \in Q}\left(\tilde{\phi}, \xi^{\prime}\right) \check{\xi}(t)\right\|_{H^{s}}(s>0)$. Let $\delta_{j}$ be the Dirac delta function at $j$. We have

$$
\begin{aligned}
& \left\|\sum_{\xi^{\prime} \in Q}\left(\tilde{\phi}, \xi^{\prime}\right) \check{\xi}(t)\right\|_{H^{s}} \\
= & {\left[\sum_{j}|j|^{2 s}\left|\sum_{\xi^{\prime} \in Q}\left(\tilde{\phi}, \xi^{\prime}\right)\left(\check{\xi}(t), \delta_{j}\right)\right|^{2}\right]^{1 / 2} } \\
= & {\left[\sum_{j}|j|^{2 s}\left|\sum_{k} \sum_{\xi^{\prime} \in Q} \hat{\phi}(k) \xi^{\prime}(k, 0)\left(\check{\xi}(t), \delta_{j}\right)\right|^{2}\right]^{1 / 2} }
\end{aligned}
$$


From the support of $\xi^{\prime}(2.25)$,

$$
|| j|-| k|| \leq 2(\log T)^{\sigma}(\sigma>2) .
$$

Since

$$
|j|>2 J_{0}=8 A(\log T)^{\sigma} \quad(A>1)
$$

from $(4.3),(4.29,4.30)$ imply

$$
|j| / 2<|k|<2|j| .
$$

We now make a dyadic decomposition of $\phi$. Let $R$ be dyadic and

$$
R / 2<|j|<2 R .
$$

So

$$
R / 4<|k|<4 R \text {. }
$$

Let

$$
\phi_{R}=\sum_{R / 4<|k|<4 R} \hat{\phi}(k) e^{i k x} .
$$

We then have

$$
\begin{aligned}
(4.28) & \leq\left[\sum_{R \text { dyadic }} 4^{s} R^{2 s} \sum_{R / 2<|j|<2 R}\left|\sum_{k} \sum_{\xi^{\prime} \in Q} \hat{\phi}_{R}(k) \xi^{\prime}(k, 0)\left(\check{\xi}(t), \delta_{j}\right)\right|^{2}\right]^{1 / 2} \\
& \leq\left[\sum_{R \text { dyadic }} 4^{s} R^{2 s}\left\|\sum_{\xi^{\prime} \in Q}\left(\tilde{\phi}_{R}, \xi^{\prime}\right) \check{\xi}(t)\right\|_{2}^{2}\right]^{1 / 2}
\end{aligned}
$$

Using (4.27) and since supp $\phi_{R} \subset \operatorname{supp} \phi \subseteq\left[-J / 2,-2 J_{0}\right] \cup\left[2 J_{0}, J / 2\right]$,

$$
\begin{aligned}
\left\|\sum_{\xi^{\prime} \in Q}\left(\tilde{\phi}_{R}, \xi^{\prime}\right) \check{\xi}(t)\right\|_{2} & \leq\left\|S(t) \phi_{R}\right\|_{2}+2 e^{-\frac{1}{2}(\log T)^{\left(\frac{\sigma^{\prime}-\delta}{\alpha}\right)}}\left\|\phi_{R}\right\|_{2} \\
& \leq 2\left\|\phi_{R}\right\|_{2} \quad\left(\sigma^{\prime}>2 \alpha+\delta>2\right) .
\end{aligned}
$$

Using (4.36) in (4.35), we have

$$
\begin{aligned}
& \left\|\sum_{\xi^{\prime} \in Q}\left(\tilde{\phi}, \xi^{\prime}\right) \check{\xi}(t)\right\|_{H^{s}} \\
\leq & {\left[\sum_{R \text { dyadic }} 4^{s} R^{2 s} \cdot 4\left\|\phi_{R}\right\|_{2}\right]^{1 / 2} } \\
\leq & C^{s}\|\phi\|_{H^{s}} .
\end{aligned}
$$

Combining (4.37) with $(4.27,4.1)$, we obtain (4.9) with a slightly larger $C$.

Proof of the Theorem. We use the decomposition in (4.4-4.6), which decomposes into low, intermediate and high frequencies. (4.9) controls (4.5), the intermediate frequencies: $2 J_{0} \leq|j| \leq J / 2,(4.7)$ controls (4.6), the high frequencies: $|j|>J / 2$. So the 
only work left is to control (4.4), the low frequencies: $|j| \leq 2 J_{0}$, which we do by iterating $S(0, T) \stackrel{\text { def }}{=} S(T),|T|$ times and each time making again the decomposition as in (4.4-4.6).

We have

$$
\begin{aligned}
& \left\|\Pi_{J / 4} S(0, t) \Pi_{2 J_{0}} u_{0}\right\|_{H^{s}} \\
\leq & \left\|\Pi_{J / 4} S(1, t) \Pi_{2 J_{0}} S(0,1) \Pi_{2 J_{0}} u_{0}\right\|_{H^{s}} \\
+ & \left\|\Pi_{J / 4} S(1, t)\left(\Pi_{J / 2}-\Pi_{2 J_{0}}\right) S(0,1) \Pi_{2 J_{0}} u_{0}\right\|_{H^{s}} \\
+ & \left\|\Pi_{J / 4} S(1, t)\left(I-\Pi_{J / 2}\right) S(0,1) \Pi_{2 J_{0}} u_{0}\right\|_{H^{s}}
\end{aligned}
$$

which is the analogue at $t=1$ of the decomposition in (4.4-4.6), with $S(0,1) \Pi_{2 J_{0}} u_{0}$ replacing $u_{0}$. So we have

$$
\begin{aligned}
(4.39) & \leq C^{s}\left\|S(0,1) \Pi_{2 J_{0}} u_{0}\right\|_{H^{s}} \\
& \leq 2 C^{2 s} s !
\end{aligned}
$$

where we used

$$
\|S(0,1)\|_{H^{s} \rightarrow H^{s}} \leq 2 C^{s} s !
$$

from (3.3) and

$$
\begin{aligned}
(4.40) & \leq\left\|S(0,1) \Pi_{2 J_{0}} u_{0}\right\|_{H^{s}} \cdot \frac{\left(C^{s} s !\right)^{4}}{J}\left(1+|T|^{3 s+1}\right) \\
& \leq 2 C^{s} s !
\end{aligned}
$$

$(4.41,4.42)$ are the analogues of $(4.9,4.7)$, which control $(4.5,4.6)$.

Using $(4.41,4.42)$, we have after one iteration:

$$
\begin{aligned}
& \left\|\Pi_{J / 4} S(0, t) \Pi_{2 J_{0}} u_{0}\right\|_{H^{s}} \\
\leq & \left\|\Pi_{J / 4} S(1, t) \Pi_{2 J_{0}} S(0,1) \Pi_{2 J_{0}} u_{0}\right\|_{H^{s}} \\
& +4 C^{2 s} s !
\end{aligned}
$$

After $r$ iterations, the analogue of the bound on (4.39) is

$$
\begin{aligned}
& C^{s}\left\|S(r, r-1) \Pi_{2 J_{0}} S(r-1, r-2) \Pi_{2 J_{0}} \cdots \Pi_{2 J_{0}} u_{0}\right\|_{H^{s}} \\
& \leq C^{s}\|S(r, r-1)\|_{H^{s} \rightarrow H^{s}} \cdot\left(2 J_{0}\right)^{s} \\
& \leq 2 C^{2 s} s !\left(2 J_{0}\right)^{s} ;
\end{aligned}
$$

while the analogue of the bound on (4.40) is

$$
2 C^{s} s !\left(2 J_{0}\right)^{s} \text {. }
$$

After $|T|$ iterations, we then have

$$
\begin{aligned}
& \left\|\Pi_{J / 4} S(0, t) \Pi_{2 J_{0}} u_{0}\right\|_{H^{s}} \\
\leq & \left\|\Pi_{2 J_{0}} S(T-1, T) \Pi_{2 J_{0}} \cdots \Pi_{2 J_{0}} S(r-1, r) \Pi_{2 J_{0}} \cdots \Pi_{2 J_{0}} S(0,1) \Pi_{2 J_{0}} u_{0}\right\|_{H^{s}} \\
& +4 C^{2 s} s !\left(2 J_{0}\right)^{s}|T| \\
\leq & |T|\left(s J_{0}\right)^{s} \cdot C^{s}
\end{aligned}
$$


with a larger $C$.

Using (4.47) in (4.4) and combining with $(4.9,4.7)$, we obtain

$$
\left\|\Pi_{J / 4} S(0, T) u_{0}\right\|_{H^{s}} \leq|T|\left(s J_{0}\right)^{s} C^{s} .
$$

Using (4.48) in (4.2), we have

$$
\left\|S(0, T) u_{0}\right\|_{H^{s}} \leq C^{s}|T|\left(s J_{0}\right)^{s}
$$

for all $0<s \leq \log T$. Interpolating with the $L^{2}$ bound $\left\|u_{0}\right\|_{L^{2}} \leq 1$ yields

$$
\begin{aligned}
\|S(0, T)\|_{H^{s^{\prime}} \rightarrow H^{s^{\prime}}} & \leq|T|^{s^{\prime} / s}\left(C s J_{0}\right)^{s^{\prime}} \\
& \leq C^{s^{\prime}}(\log T)^{(\sigma+1) s^{\prime}} \quad(\sigma>2)
\end{aligned}
$$

with a larger $C$, for all $0<s^{\prime}<s$, where we took $s=\log |T|$ and used (4.3).

For a fixed $s>0$, for $|t|<e^{s}$, the a priori bound (3.3) gives

$$
\|S(0, t)\|_{H^{s} \rightarrow H^{s}} \leq C^{s} s ! e^{s^{2}}
$$

for $|t| \geq e^{s}$, we use (4.50). This gives immediately

$$
\|S(0, t)\|_{H^{s} \rightarrow H^{s}} \leq C_{s}(\log (|t|+2))^{(\sigma+1) s}
$$

for all $s>0$. Let $\varsigma=\sigma+1$, we obtain the theorem.

\section{REFERENCES}

[B1] J. Bourgain, Growth of Sobolev norms in linear Schrödinger operators with quasi-periodic potential, Commun. Math. Phys. (1999), 207-247.

[B2] J. Bourgain, Global solutions of nonlinear Schrödinger equations, Colloquim Publication, AMS, 1999.

[B3] J. Bourgain, On the growth of Sobolev norms in linear Schrödinger operators with smooth time dependent potential, J. Anal. Math. 77 (1999), 315-348.

[Hö] L. Hörmander, Analysis of linear partial differential operators I, II, Springer-Verlag, 1990.

[N] V. Nersesyan, Growth of Sobolev norms and controllability of Schrödinger equation, ArXiv: 0804.3982v2 (2008).

[W] W.-M. Wang, Bounded Sobolev norms for linear Schrödinger equations under resonant perturbations, J. Func. Anal. 254 (2008), 2926-2946.

Departement de Mathematique, Universite Paris Sud, 91405 Orsay Cedex, FRAnCE

E-mail address: wei-min.wang@math.u-psud.fr 